\title{
Erratum: Particle-tracking model of outfall plumes in a tidal channel
}

S. Samuel Li PhD, PEng, MIAHR Associate Professor in Hydrotechnical Engineering, Department of Building, Civil and Environmental Engineering, Concordia University, Montreal, Canada

\section{Song Liu MASC}

Graduate Student, Department of Building, Civil and Environmental

Engineering, Concordia University, Montreal, Canada

The publisher regrets that the following errors appeared in this paper when it was published in Water Management 166(4): 175-186.

Equation 1 was published as

1. $\boldsymbol{r}_{i}(t+\Delta t)=\boldsymbol{r}_{i}(t)+\boldsymbol{v}\left(\boldsymbol{r}_{i}, t\right) \Delta t+\boldsymbol{r}_{i}(t)$

It should be

1. $\boldsymbol{r}_{i}(t+\Delta t)=\boldsymbol{r}_{i}(t)+\boldsymbol{v}\left(\boldsymbol{r}_{i}, t\right) \Delta t+\boldsymbol{r}_{i}^{\prime}(t)$ 